\title{
Aceitação sensorial de bebidas lácteas potencialmente simbióticas
}

\author{
Sensory acceptance of potentially symbiotic dairy beverages
}

\section{Autores | Authors \\ 凶 Janaína Fernandes de Medeiros BURKERT \\ Universidade Federal do Rio Grande (FURG) \\ Escola de Química e Alimentos Laboratório de Análise Sensorial e Controle de Qualidade Caixa Postal: 474 CEP: $96201-900$ Rio Grande/RS - Brasil e-mail: jfmb@vetorial.net}

Renata Aline dos Santos da FONSECA

Jaqueline Oliveira de MORAES Janaína SGANZERLA

Universidade Federal do Rio Grande (FURG)

Escola de Química e Alimentos Laboratório de Análise Sensorial e Controle de Qualidade Rio Grande/RS - Brasil

e-mail: re_aline@ibest.com.br jaquelinenemoraes111@gmail.com jana.ea@hotmail.com

Susana Juliano KALIL

Universidade Federal do Rio Grande (FURG)

Escola de Química e Alimentos Laboratório de Microbiologia Rio Grande/RS - Brasil e-mail:dqmsjk@furg.br

\section{Carlos André Veiga BURKERT}

Universidade Federal do Rio Grande (FURG) Escola de Química e Alimentos Laboratório de Engenharia de Bioprocessos Rio Grande/RS - Brasil e-mail: burkert@vetorial.net

$\square$ Autor Correspondente / Corresponding Author

Recebido / Received: 13/10/2010 Aprovado / Approved: 08/08/2012 Publicado / Published: dez./2012

\section{Resumo}

A procura por alimentos funcionais, como leites fermentados com culturas microbianas probióticas, aumentou o interesse em pesquisas para manter estes micro-organismos viáveis no produto. Neste sentido, o objetivo deste trabalho foi formular um leite fermentado sabor morango agregado de benefícios potencialmente prebióticos e probióticos, apresentando características sensoriais adequadas. Um planejamento fatorial $2^{3}$ com ponto central foi realizado para otimizar a saborização do produto, avaliando a adição de sacarose (50, 85 e $\left.120 \mathrm{~g} . \mathrm{L}^{-1}\right)$, corante natural carmim cochonilha $\left(0,5 ; 1,0\right.$ e 1,5 g. $\left.\mathrm{L}^{-1}\right)$ e aroma natural de morango $\left(0,10 ; 0,25\right.$ e 0,40 g. $\left.\mathrm{L}^{-1}\right)$. Diferentes concentrações da cultura Bio Rich ( $L$. acidophilus, Bifidobacterium e $S$. thermophilus) foram testadas (1,00; 0,50 e 0,33 g. $\left.\mathrm{L}^{-1}\right)$, ordenando-se sensorialmente conforme a preferência. Concentrações do xarope de fruto-oligossacarídeos (50 e $100 \mathrm{~g} . \mathrm{L}^{-1}$ ) foram avaliadas por meio de aceitação sensorial e análises microbiológicas. Um leite fermentado sabor morango utilizando, em g.L $\mathrm{L}^{-1}$, 0,33 de cultura, 120 de sacarose, 1,5 de corante, 0,4 de aroma e 100 de xarope de fruto-oligossacarídeos, foi desenvolvido. 0 incremento da concentração de prebiótico influenciou positivamente na preferência do produto, apresentando índice de aceitação de 88\%; microbiologicamente, aumentou a contagem de $L$. acidophilus e Bifidobacterium, sendo $1,7 \times 10^{7} \mathrm{e}$ 4,8 × $10^{6}$ UFC. $\mathrm{mL}^{-1}$, respectivamente, com $100 \mathrm{~g} \cdot \mathrm{L}^{-1}$ de prebiótico.

Palavras-chave: Desenvolvimento de produtos; Prebióticos; Probióticos.

\section{Summary}

The demand for functional foods, including milk fermented with probiotic microbial cultures, has increased the interest in research to maintain these microorganisms viable in the product. Thus the objective of this work was to formulate strawberry flavoured fermented milk with added potentially beneficial prebiotics and probiotics, and with suitable sensory characteristics. A $2^{3}$ experimental design was used to optimize the product flavour, evaluating the addition of sucrose $\left(50,85\right.$ and $\left.120 \mathrm{~g} \cdot \mathrm{L}^{-1}\right)$, the natural dye cochineal carmine $\left(0.5,1.0\right.$ and $\left.1.5 \mathrm{~g} . \mathrm{L}^{-1}\right)$ and a natural strawberry aroma $\left(0.10,0.25\right.$ and $\left.0.40 \mathrm{~g} . \mathrm{L}^{-1}\right)$. Different concentrations of Bio Rich culture (L. acidophilus, Bifidobacterium and $S$. thermophilus) were tested $\left(1.00,0.50\right.$ and $\left.0.33 \mathrm{~g} . \mathrm{L}^{-1}\right)$ ranking the products in according to sensory preference. Two fructo-oligosaccharide syrup concentrations (50 and $100 \mathrm{~g} . \mathrm{L}^{-1}$ ) were evaluated according to their sensory acceptability and microbiological analyses. A strawberry flavoured fermented dairy beverage was developed using (g.. $\left.\mathrm{L}^{-1}\right) 0.33$ culture, 120 sucrose, 1.5 dye, 0.4 aroma and 100 fructo-oligosaccharide syrup. The increase in concentration of the prebiotics positively influenced product preference, with acceptance rates of $88 \%$ and increased microbiological counts of $L$. acidophilus and Bifidobacterium at $1.7 \times 10^{7}$ and $4.8 \times 10^{6}$ CFU. $\mathrm{mL}^{-1}$ for $100 \mathrm{~g} . \mathrm{L}^{-1}$ of prebiotics.

Key words: Product development; Prebiotics; Probiotics. 


\section{Introdução}

Consumidores preocupados com a manutenção da saúde e do bem-estar procuram alimentos com características de qualidade que aportem ganhos fisiológicos, além de requerimentos sensoriais e nutricionais básicos. Esta crescente busca por uma alimentação equilibrada tem modificado o entendimento do papel da dieta sobre a saúde e incentivado o estudo de componentes naturais biologicamente ativos. Neste sentido, é de interesse dos pesquisadores o desenvolvimento de novos produtos lácteos que desempenhem, além das funções de nutrição e de prover apelo sensorial, uma terceira função com efeitos benéficos; esses produtos são conhecidos como alimentos funcionais, em que se destacam os probióticos e os prebióticos (HASLER et al., 2004; THAMER e PENNA, 2006; VILELA, 2002; MARTINS e BURKERT, 2009).

De acordo com o Regulamento Técnico de Substâncias Bioativas e Probióticos Isolados com Alegação de Propriedades Funcionais e/ou de Saúde, Resolução $\mathrm{RDC} \mathrm{n}^{\circ} 2$ de janeiro de 2002, entende-se por probióticos os micro-organismos vivos capazes de melhorar o equilíbrio microbiano intestinal, produzindo efeitos benéficos à saúde do indivíduo (BRASIL, 2002). Atuam ativando os processos metabólicos, melhorando as condições de saúde pelo aumento da efetividade do sistema imune e prevenindo o aparecimento precoce de alterações patológicas e doenças degenerativas, que levam à redução da longevidade (CHOCKCHAISAWASDEE et al., 2005; THAMER e PENNA, 2006).

Os probióticos são micro-organismos vivos que beneficiam o hospedeiro por melhorar seu equilíbrio intestinal. Os mais conhecidos são as bactérias lácticas e bifidobactérias, que são largamente usados em iogurte e outros derivados do leite. Esses organismos não são patógenos nem toxigênicos, e devem sobreviver ao processo de armazenamento e à passagem pelo sistema digestivo, além de produzir efeitos benéficos, como a manutenção normal da microbiota intestinal, ter atividade anticarcinogênica, estímular o sistema imunológico e melhorar o valor nutricional dos alimentos (CRITTENDEN e PLAYNE, 1996; MARX et al., 2000).

Os prebióticos são considerados alimentos não digeríveis que beneficiam o hospedeiro ao estimular, seletivamente, o crescimento e/ou a atividade das bactérias "benéficas", resistentes no cólon intestinal, tendo efeito antagônico ao desenvolvimento de microorganismos patogênicos e diminuindo, dessa forma, os compostos tóxicos (CHOCKCHAISAWASDEE et al., 2005). Dentre estes prebióticos, está uma das maiores classes de oligossacarídeos bifidogênicos, os frutooligossacarídeos (FOS). O principal interesse na utilização destes como ingredientes nos alimentos é em virtude das suas propriedades fisiológicas (CRITTENDEN e PLAYNE, 1996).

A análise sensorial definida pela ISO (International Standard Organization) como "o exame das propriedades sensoriais de um produto através dos órgãos dos sentidos" é um instrumento fundamental para determinar o grau de aceitação do produto pelo consumidor (PIANA et al., 2004). A ferramenta da Metodologia de Superfície de Resposta (MSR) é muito utilizada na otimização de diversos processos em alimentos, no desenvolvimento de novos produtos e, em análise sensorial, fornece resultados que indicam a melhor formulação de diferentes produtos (QUEIROZ et al., 2003; VALIM et al., 2003; MORAIS et al., 2008; CASTRO et al., 2009; OLIVEIRA e JURKIEWICZ, 2009).

Dessa forma, o presente trabalho teve por objetivo desenvolver um leite fermentado, potencialmente simbiótico, adicionado de FOS e probióticos, avaliando a saborização do produto, a viabilidade dos microorganismos probióticos e a aceitação do produto final.

\section{Material e métodos}

\subsection{Leite fermentado}

Para a obtenção do leite fermentado, foram utilizados: $1000 \mathrm{~mL}$ de leite U.H.T. integral (Danby Cosulati), $50 \mathrm{~g}$ de leite em pó desnatado instantâneo (Elegê) e $1 \mathrm{~g}$ de cultura láctea (descrita e selecionada no item 2.2); estes ingredientes foram misturados e incubados em banho termostatizado a $42{ }^{\circ} \mathrm{C}$ para $\mathrm{o}$ processo de fermentação, que foi considerado completo quando o pH atingiu 4,6 (CUNHA et al., 2009). A acidez titulável foi determinada durante o processo. Após a fermentação, o produto foi submetido a um banho de gelo para reduzir a temperatura imediatamente, sendo então acondicionado sob refrigeração $\left(5^{\circ} \mathrm{C}\right)$ durante 12 horas.

\subsection{Seleção de culturas lácteas}

Duas formulações de leite fermentado foram obtidas conforme o item 2.1, utilizando as culturas comerciais (Christian Hansen Ind. e Com. Ltda.) que apresentavam diferentes micro-organismos: uma cultura constituída de partes iguais de L. delbrueckii ssp. bulgaricus e S. thermophilus (Rich) e outra cultura descrita como probiótica, formada por L. acidophilus LA-5, Bifidobacterium sp. BB-12 e S. thermophilus (Bio Rich). Nesta etapa, as bebidas lácteas foram saborizadas com a adição de: 100 g.L $\mathrm{L}^{-1}$ de sacarose; quantidades máximas recomendadas pelos fabricantes de $50 \mathrm{~g} . \mathrm{L}^{-1}$ de polpa de fruta (Mais Fruta) sabor morango; 1,5 g.L $\mathrm{L}^{-1}$ de corante natural carmim de cochonilha (Christian Hansen Ind. e Com. Ltda.), e 0,4 g. L $^{-1}$ de aroma natural de morango (Duas Rodas Industrial Ltda.). As duas bebidas 
lácteas foram testadas por um grupo de 33 provadores não treinados, que avaliaram a influência da cultura láctea por meio da preferência, utilizando o teste de comparação pareada (ABNT, 1994a; MEILGAARD et al., 1999).

\subsection{Efeito da sacarose, aroma e corante na saborização do produto}

Com a finalidade de otimizar a saborização do produto, foi realizada a fermentação, conforme item 2.1, com a cultura probiótica Bio Rich, e foi-se avaliando sensorialmente a variável resposta aceitação (Y). Ao produto fermentado, foi acrescentada a polpa de fruta (50 g.L-1) e as variáveis independentes - concentração de sacarose $\left(X_{1}\right)$, corante $\left(X_{2}\right)$ e aroma $\left(X_{3}\right)$, apresentadas na Tabela 1 - foram estudadas por meio de um planejamento experimental $2^{3}$ fornecendo nove formulações diferentes com repetição no ponto central, totalizando 11 ensaios. Os resultados foram tratados utilizando-se a metodologia de Análise de Superfície de Resposta no software Statistica versão 5.0. As formulações foram submetidas à avaliação empregando-se uma escala hedônica de nove pontos (1-desgosto extremamente; 9-gosto extremamente), com as amostras distribuídas em delineamento de blocos incompletos, em que foi avaliada a aceitação por um de grupo de 19 provadores (STONE e SIDEL, 1985).

\subsection{Fermentações com diferentes concentrações de culturas}

A fim de selecionar a concentração da cultura Bio Rich, foi usado um teste de ordenação (ABNT, 1994b; MEILGAARD et al., 1999), com 33 provadores, que avaliaram a preferência entre três bebidas lácteas fermentadas com diferentes concentrações da cultura Bio Rich (0,33; 0,50 e 1,00 g. $\left.\mathrm{L}^{-1}\right)$, sendo saborizadas com a condição previamente selecionada, por meio do estudo no planejamento fatorial.

\subsection{Adição de fruto-oligossacarídeos na fermentação}

Aos ingredientes para obtenção do leite fermentado natural (obtido de acordo com item 2.1), foi adicionado xarope de FOS (Nutraflora ${ }^{\circledR}$ ), gentilmente cedido pela Corn Products, em diferentes concentrações: sem adição de FOS, adicionado de $50 \mathrm{~g} \cdot \mathrm{L}^{-1}$ e $100 \mathrm{~g} \cdot \mathrm{L}^{-1}$ de FOS. As amostras foram inoculadas com 0,33 g. $\mathrm{L}^{-1} \mathrm{da}$ cultura Bio Rich. Após a fermentação, as bebidas foram saborizadas conforme as condições otimizadas no item 2.3 e submetidas a um teste de diferença do controle (ABNT, 1995; MEILGAARD et al., 1999), utilizando-se um padrão - sem FOS - para avaliar a concentração de FOS, com um grupo de 51 provadores e uma escala em que 1-Extremamente melhor que o padrão e 9-Extremamente inferior ao padrão, sendo 5-Nenhuma diferença do padrão.

\subsection{Análises físico-químicas}

\subsection{Determinação do $\mathrm{pH}$}

A determinação do $\mathrm{pH}$ foi realizada por meio de leitura em potenciômetro digital (IAL, 1985).

\subsubsection{Determinação da acidez titulável}

As determinações dos teores de acidez foram realizadas pela titulação ácido-alcalimétrica, utilizando-se fenolftaleína como indicador (IAL, 1985).

\subsection{Análise sensorial}

Para a análise sensorial, foram utilizadas amostras de $25 \mathrm{~mL}$ de leite fermentado a $5{ }^{\circ} \mathrm{C}$, em copos descartáveis codificados com números aleatórios de três dígitos (STONE e SIDEL, 1985). Todos os julgadores pertenciam à comunidade acadêmica.

Tabela 1. Médias para aceitação das bebidas lácteas saborizadas por meio do planejamento experimental $2^{3}$.

\begin{tabular}{|c|c|c|c|c|c|c|c|}
\hline \multirow{2}{*}{ Ensaio } & \multicolumn{3}{|c|}{ Valores codificados } & \multicolumn{3}{|c|}{ Valores reais $\left(g \cdot \mathrm{L}^{-1}\right)$} & \multirow{2}{*}{ Aceitação (Y) } \\
\hline & $X_{1}$ & $X_{2}$ & $\mathrm{X}_{3}$ & Sacarose & Corante & Aroma & \\
\hline 1 & -1 & -1 & -1 & 50 & 0,5 & 0,1 & 5,75 \\
\hline 2 & +1 & -1 & -1 & 120 & 0,5 & 0,1 & 8,25 \\
\hline 3 & -1 & +1 & -1 & 50 & 1,5 & 0,1 & 5,50 \\
\hline 4 & +1 & +1 & -1 & 120 & 1,5 & 0,1 & 8,00 \\
\hline 5 & -1 & -1 & +1 & 50 & 0,5 & 0,4 & 6,25 \\
\hline 6 & +1 & -1 & +1 & 120 & 0,5 & 0,4 & 7,25 \\
\hline 7 & -1 & +1 & +1 & 50 & 1,5 & 0,4 & 6,50 \\
\hline 8 & +1 & +1 & +1 & 120 & 1,5 & 0,4 & 8,25 \\
\hline 9 & 0 & 0 & 0 & 85 & 1,0 & 0,25 & 7,00 \\
\hline 10 & 0 & 0 & 0 & 85 & 1,0 & 0,25 & 7,00 \\
\hline 11 & 0 & 0 & 0 & 85 & 1,0 & 0,25 & 7,10 \\
\hline
\end{tabular}

$\mathrm{X}_{1}$ : Sacarose; $\mathrm{X}_{2}$ : Corante natural; $\mathrm{X}_{3}$ : Aroma natural; $Y$ : Aceitação. 
No produto final obtido, foi realizado um teste de aceitação global com cem consumidores, utilizando-se escala hedônica de nove pontos, sendo o índice de aceitação calculado segundo Queiroz e Treptow (2006).

\subsection{Análises microbiológicas}

As contagens de micro-organismos (VINDEROLA e REINHEIMER, 1999) foram realizadas em duplicata nas bebidas lácteas com diferentes concentrações de FOS (sem adição, 50 e $100 \mathrm{~g} \cdot \mathrm{L}^{-1}$ ) 12 horas após a fermentação. Amostras de $25 \mathrm{~mL}$ foram homogeneizadas em $225 \mathrm{~mL}$ de citrato de sódio $2 \%$ e, em seguida, diluições decimais seriadas foram realizadas, utilizando-se o mesmo diluente. A enumeração de bactérias láticas Lactobacillus acidophilus foi realizada em meio Agar de Man, Rogosa e Sharpe (MRS), mais uma sobrecamada sendo incubada em aerobiose por 72 horas a $37^{\circ} \mathrm{C}$. Para contagem seletiva de Bifidobactérias, foi utilizado o meio LP-MRS (cloreto de lítio 0,2\% e propionato de sódio 0,3\%), sendo incubado em anaerobiose (Anaerocult A) nas mesmas condições.

\subsection{Análises estatísticas}

Para tratamento dos dados de análise sensorial utilizando-se o teste de comparação pareada (item 2.2), foi aplicada a tabela de Roessler $(p \leq 0,05)$ (ABNT, 1994a; MEILGAARD et al., 1999). Os resultados do teste de ordenação (item 2.4) foram analisados pelo teste de Friedman $(p \leq 0,05)$ usando-se a tabela de Newell e MacFarlane (ABNT, 1994b; MEILGAARD et al., 1999) e, para o teste de diferença do controle (item 2.5), foi aplicada a Análise de Variância seguida do teste de Dunnett $(p \leq 0,05)$ (ABNT, 1995; MEILGAARD et al., 1999).

A aceitação das diferentes formulações (STONE e SIDEL, 1985) obtidas por meio do planejamento fatorial completo $2^{3}$ (item 2.3) foi tratada utilizando-se o software Statistica versão 5.0 e verificando-se a Análise de Variância e o teste de Fisher ( $p \leq 0,05)$, para obtenção de um modelo empírico de primeira ordem e a construção das superfícies de resposta (RODRIGUES e IEMMA, 2009).

Para as análises microbiológicas, foi realizado o tratamento estatístico do teste t de Student $(p \leq 0,05)$, segundo Gomes (2000).

\section{Resultados e discussão}

\subsection{Influência da cultura láctea na preferência do leite fermentado}

Em todas as fermentações realizadas, o tempo final de processo foi de aproximadamente 4,6 horas, para alcançar $\mathrm{pH}$ 4,6 com cerca de $9^{\circ} \mathrm{D}$ de ácido lático. Para a seleção da cultura probiótica, foi realizado o teste sensorial de comparação pareada, na qual 15 dos 33 julgadores preferiram a amostra Bio Rich e 18 julgadores, a Rich. A partir da tabela de Roessler $(p \leq 0,05)$, seria necessário que pelo menos 23 julgadores preferissem uma amostra para que houvesse diferença significativa. Portanto, como não houve diferença significativa a 5\% de significância, a cultura descrita como probiótica Bio Rich (item 2.2.) foi selecionada para a sequência do estudo da saborização do leite fermentado com potencial simbiótico.

\subsection{Saborização do leite fermentado}

Por meio do planejamento experimental $2^{3}$, foram saborizadas nove bebidas lácteas diferentes, com repetições no ponto central, totalizando 11 ensaios, cujas formulações com as respectivas médias para a aceitação estão descritas na Tabela 1.

De acordo com a análise de variância (ANOVA) exposta na Tabela 2, foi possível obter um modelo de primeira ordem capaz de prever a aceitação em função das concentrações de sacarose, corante e aroma.

Para a aceitação global do produto - que considera todos os atributos sensoriais, como gosto, sabor, aroma e parâmetros de textura - por meio da ANOVA, é possível verificar que o coeficiente de determinação $r^{2}=0,99 \mathrm{e}$ a razão $\mathrm{Fcal} / \mathrm{Ftab}=11,77$ demonstraram que o modelo codificado representado na Equação 1 é considerado preditivo e significativo $(p \leq 0,05)$. Consequentemente, a partir deste modelo codificado, foram obtidas as curvas de contorno apresentadas na Figura 1.

$$
\begin{aligned}
& Y=6,98+0,97 X_{1}+0,09 X_{2}+0,09 X_{3}+ \\
& +0,09 X_{1} X_{2}-0,28 X_{1} X_{3}+0,22 X_{2} X_{3}
\end{aligned}
$$

em que: $Y=$ aceitação; $X_{1}, X_{2}$ e $X_{3}=$ variáveis codificadas estudadas: sacarose, corante natural e aroma natural, respectivamente.

Na Figura 1, pode ser observado que, independentemente do nível de concentração de corante utilizado, o aumento da concentração de açúcar aumentou a aceitação do produto (Figura 1a), e os escores máximos foram alcançados em torno de $120 \mathrm{~g} \cdot \mathrm{L}^{-1}$

Tabela 2. Análise de variância para aceitação de bebidas lácteas.

\begin{tabular}{lcccc}
$\begin{array}{l}\text { Fonte de } \\
\text { variação }\end{array}$ & $\begin{array}{c}\text { Soma } \\
\text { quadrática }\end{array}$ & $\begin{array}{c}\text { Graus de } \\
\text { liberdade }\end{array}$ & $\begin{array}{c}\text { Média } \\
\text { quadrática }\end{array}$ & $\mathbf{F}_{\text {calculado }}$ \\
Regressão & 8,73 & 6 & 1,45 & 72,50 \\
Resíduo & 0,09 & 4 & 0,02 & \\
Falta de & 0,08 & 2 & & \\
ajuste & & & & \\
Erro puro & 0,01 & 2 & & \\
Total & 8,82 & 10 & & \\
\hline
\end{tabular}

$r^{2}=0,99 ; F_{(0,95 ; 6 ; 4)}=6,16$. 
de sacarose. Comportamento similar foi observado com a concentração de aroma e sacarose (Figura 1b), obtendo os melhores valores ao redor de $120 \mathrm{~g} \cdot \mathrm{L}^{-1}$ de sacarose. Na Figura 1c, é possível verificar que a maior aceitação sensorial do produto foi obtida na região em torno das maiores concentrações de aroma $0,4 \mathrm{~g} \cdot \mathrm{L}^{-1}$ e das maiores concentrações de corante $1,5 \mathrm{~g} \cdot \mathrm{L}^{-1}$, demonstrando que, apesar de o aumento da concentração de sacarose intensificar a aceitação pelas bebidas lácteas, independentemente dos teores de aroma e corante, a interação entre os mesmos mostra a tendência de que os valores maiores incrementam positivamente na resposta sensorial avaliada.

Portanto, por meio da análise das curvas de contorno para aceitação do produto, foi definido como as melhores condições, na faixa estudada, para saborização do leite fermentado sabor morango, a adição de $120 \mathrm{~g} \cdot \mathrm{L}^{-1}$ de sacarose, 1,5 g...-1 de corante e 0,4 g.L $\mathrm{L}^{-1}$ de aroma.

Villegas et al. (2009) também utilizaram uma estratégia de aplicação de Metodologia de Superfície de Resposta semelhante a este trabalho para o desenvolvimento de bebidas lácteas desnatadas sabor baunilha, utilizando dois tipos do prebiótico inulina, variando a sua concentração $(30-80$ g.L-1 $)$ e de sacarose (0-80 g.L-1), para avaliar a aceitação sensorial; porém, por meio dos modelos e superfícies, verificaram que não houve diferença entre as formulações das bebidas com relação ao tipo e à concentração de inulina.

No estudo da adição de prebióticos em leites fermentados probióticos, um planejamento experimental foi realizado, em que as variáveis independentes foram a goma acácia (0 a 3\%) e a inulina (0 a 3\%), estudadas em cinco níveis, resultando em 11 ensaios. Estes demonstraram, por meio da aceitação sensorial com escala hedônica de nove pontos, que não houve diferença significativa $(p>0,05)$ entre essas variáveis (OLIVEIRA e JURKIEWICZ, 2009).

\subsection{Influência da concentração da cultura láctica na preferência do leite fermentado}

O teste de Friedman, utilizando a tabela de Newel e McFarlane, indica a diferença crítica entre os totais de ordenação, de acordo com o número de tratamentos testados e o número de julgamentos obtidos (QUEIROZ e TREPTOW, 2006). Os totais de ordenação para as três amostras foram obtidos com base na somatória das notas dadas pelos provadores. As formulações contendo 0,33, 0,5 e 1,0 g. L ${ }^{-1}$ de cultura Bio Rich tiveram, como totais de ordenação, respectivamente, 68,69 e 61 . O limite crítico para o número de provadores participantes do teste de ordenação, segundo a tabela de Newel e McFarlane, é 20 , por meio do qual se sabe se há diferença significativa entre as amostras em nível de significância de 5\% (QUEIROZ e TREPTOW, 2006). O módulo da diferença entre os totais das formulações contendo 0,33 e $0,5 \mathrm{~g} \cdot \mathrm{L}^{-1}$ foi a unidade (1), enquanto os módulos das diferenças entre os totais das formulações com 0,33 e 1,0 g.L-1 e as formulações com 0,5 e 1,0 g.L $\mathrm{L}^{-1}$ da cultura Bio Rich foram 7 e 8 , respectivamente. Com base nesses resultados, as amostras não diferem significativamente entre si, em nível de 5\%; deste modo, a menor concentração da cultura láctica estudada, de $0,33 \mathrm{~g} . \mathrm{L}^{-1}$, foi selecionada para obtenção do produto, visando a minimizar custos.

Olson e Aryana (2008) estudaram diferentes concentrações de $L$. acidophilus $(0 ; 0,0239 ; 0,238$ e 2,33 g) em $100 \mathrm{~g}$ de bebidas lácteas e verificaram, sensorialmente, por meio de testes de escalas, que não houve diferença para o aroma e para a textura e a aparência; apenas a amostra com maior quantidade do probiótico apresentou menor pontuação em razão da alta concentração do mesmo, diferindo das demais $(p \leq 0,05)$, indicando que a concentração de micro-organismos starter não interfere em alguns parâmetros sensoriais desde que não seja considerada excessivamente elevada, como demonstraram os autores. (a)

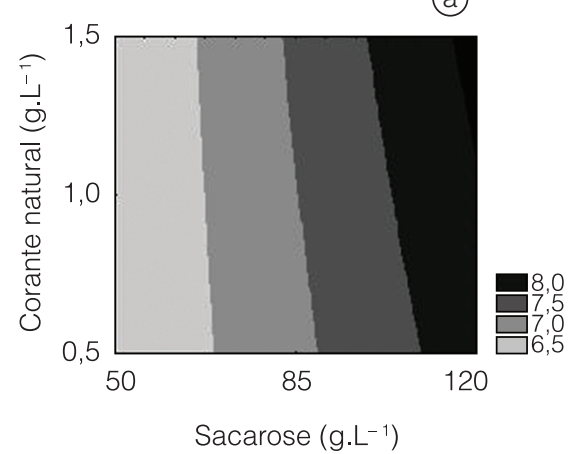

(b)

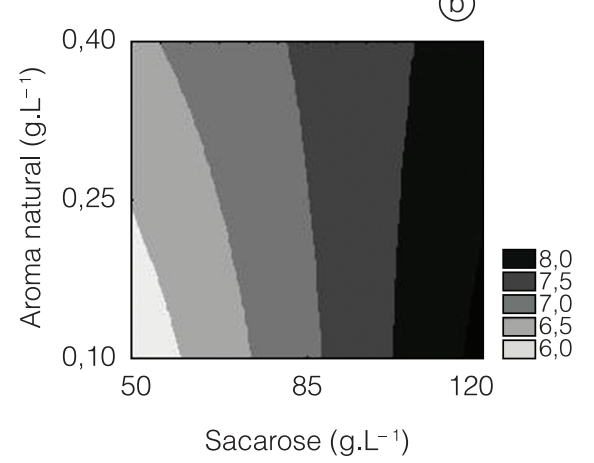

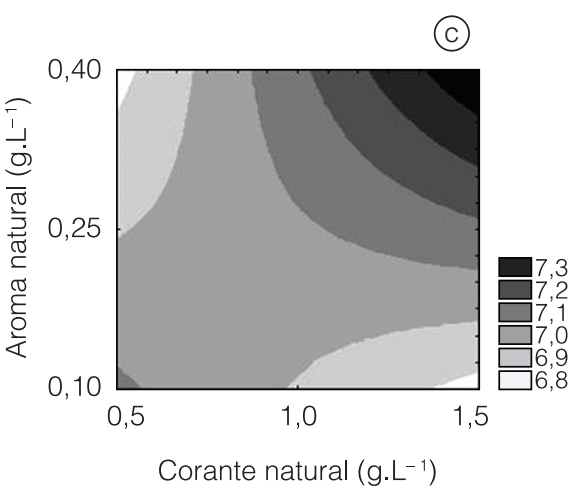

Figura 1. Curvas de Contorno para aceitação das bebidas lácteas em função da concentração de corante e sacarose com aroma fixado em 0,25 g.L $\mathrm{L}^{-1}$ (a); concentração de aroma e sacarose com corante mantido em $1 \mathrm{~g} \cdot \mathrm{L}^{-1}$ (b), e concentração de corante e aroma com sacarose fixa em $85 \mathrm{~g} \cdot \mathrm{L}^{-1}(\mathrm{c})$. 
No trabalho de Mazochi et al. (2010), foram desenvolvidos iogurtes de leite de cabra contendo ou não B. longum, B. breve, B. pseudolongum e B. bifidum, adicionados ou não de aroma de morango que, submetidos a testes de escala hedônica, demonstraram que a adição de diferentes micro-organismos probióticos não apresentou diferenças entre esses iogurtes para os atributos aparência, aroma, sabor e textura.

\subsection{Influência da concentração de FOS}

Bebidas lácteas fermentadas com a adição do ingrediente prebiótico xarope de FOS, nas concentrações de 50 e $100 \mathrm{~g} \cdot \mathrm{L}^{-1}$, foram comparadas com a bebida controle (sem FOS), utilizando-se um teste de diferença do controle. As bebidas lácteas controle, 50 e 100 g.L L $^{-1}$ de FOS obtiveram, respectivamente, as médias de $4,59^{a} ; 4,47^{a}$ e $3,67^{b}$, demonstrando que a adição de até 50 g.L $\mathrm{L}^{-1}$ do FOS não apresentou diferença significativa $(p>0,05)$ em relação ao controle. No entanto, a adição de $100 \mathrm{~g} . \mathrm{L}^{-1}$ apresentou diferença significativa entre moderadamente e ligeiramente melhor do que o controle, conforme a escala sensorial utilizada, sendo esta amostra selecionada para avaliação da aceitação do produto com prováveis características simbióticas. Segundo a Agência Nacional de Vigilância Sanitária (BRASIL, 2005), para alimentos líquidos, o teor requerido é de 1,5 g, não devendo ultrapassar $30 \mathrm{~g}$ na recomendação diária do produto pronto para consumo, quantidade esta também indicada pelo fabricante do produto.

Castro et al. (2008) pesquisaram a influência da adição de oligofrutose (0, 2 e 5\%) em bebidas lácteas ABT (Lactobacillus acidophilus, Bifidobacterium ssp. e Streptococcus salivarius ssp. thermophilus) com $30 \%$ de soro de queijo, demonstrando que a adição do prebiótico contribuiu positivamente nas características sensoriais, diferindo da amostra sem a presença do mesmo.

\subsection{Teste de aceitação global do produto final}

$O$ produto final obtido de $1 \mathrm{~L}$ de leite fermentado, com $50 \mathrm{~g}$ de leite em pó e 0,33 g. $\mathrm{L}^{-1}$ de cultura Bio Rich, saborizado com 50 g. $\mathrm{L}^{-1}$ de polpa de fruta sabor morango, $120 \mathrm{~g} \cdot \mathrm{L}^{-1}$ de sacarose, $1,5 \mathrm{~g} \cdot \mathrm{L}^{-1}$ de corante natural carmin de cochonilha e 0,4 g. $\mathrm{L}^{-1}$ de aroma natural de morango, e adicionado de $10 \%$ de xarope de FOS, apresentou um índice de aceitação de $88 \%$. Borges et al. (2009) encontraram índices de aceitação em torno de $80 \%$ para iogurtes elaborados com leite de búfala sabor cajá. Ambos os resultados estão de acordo com a aceitação mínima que um produto deve ter, ou seja, igual ou superior a $70 \%$ (TEIXEIRA, 1987).

\subsection{Análises microbiológicas}

Na Tabela 3, estão apresentadas as contagens de micro-organismos nas bebidas elaboradas com diferentes concentrações de FOS estudadas.
Tabela 3. Contagem de micro-organismos para diferentes concentrações de FOS.

\begin{tabular}{ccc}
$\begin{array}{c}\text { Concentração } \\
\text { de FOS }\end{array}$ & \multicolumn{2}{c}{$\begin{array}{c}\text { Log } \\
\text { UFC. } L^{-1}\end{array}$} \\
g. L $^{-1}$ & L. acidophilus & Bifdobacterium ssp. \\
0 & $6,36^{\mathrm{c}}$ & $5,58^{\mathrm{c}}$ \\
50 & $7,08^{\mathrm{b}}$ & $5,83^{\mathrm{b}}$ \\
100 & $7,23^{\mathrm{a}}$ & $6,68^{\mathrm{a}}$ \\
\hline
\end{tabular}

Letras distintas na coluna significam que os valores são diferentes entre si pelo teste $t$ de Student $(p \leq 0,05)$.

Ao observar a Tabela 3, pode-se verificar que, com o aumento da concentração do FOS adicionado no processo fermentativo, houve um aumento no crescimento dos micro-organismos ao término do processo de fermentação, tanto para lactobacilos quanto para bifidobactérias. Portanto, a adição do ingrediente prebiótico xarope de FOS permitiu um incremento que difere estatisticamente das demais amostras na contagem microbiana, em termos de unidades formadoras de colônia (log UFC. $\mathrm{mL}^{-1}$ ) da cultura probiótica Bio-Rich, obtendo-se, dessa forma, um produto potencialmente simbiótico.

Aryana e McGrew (2007) avaliaram a viabilidade do Lactobacillus casei em iogurtes adicionados de 1,5\% de prebióticos com diferentes graus de polimerização (pequeno grau de polimerização - oligofrutose; médio grau de polimerização - inulina GR, e grande grau de polimerização - inulina HP) frente a um iogurte controle, que forneceu uma contagem de 7,18 log UFC. $\mathrm{mL}^{-1}$, enquanto os iogurtes adicionados de prebióticos apresentaram uma contagem de 7,51.

\section{Conclusões}

Foi possível elaborar um leite fermentado sabor morango utilizando como ingredientes leite, leite em pó e 0,33 g. $\mathrm{L}^{-1}$ de cultura Bio Rich, sendo saborizado com $50 \mathrm{~g} . \mathrm{L}^{-1}$ de polpa de fruta sabor morango, $120 \mathrm{~g} . \mathrm{L}^{-1}$ de sacarose, 1,5 g. $\mathrm{L}^{-1}$ de corante natural carmin de cochonilha e 0,4 g. $\mathrm{L}^{-1}$ de aroma natural de morango, enriquecido com $10 \%$ de xarope de fruto-oligossacarídeos, sendo o índice de aceitação deste produto de $88 \%$. A contagem de L. acidophilus foi de 7,23 log UFC. $\mathrm{mL}^{-1} \mathrm{e}$, de bifidobactérias, 6,68 log UFC. $\mathrm{mL}^{-1}$.

\section{Agradecimentos}

Ao CNPq (Conselho Nacional de Desenvolvimento Científico e Tecnológico), pela concessão de Bolsa PIBICCNPq-FURG.

À FAPERGS (Fundação de Amparo à Pesquisa do Estado do Rio Grande do Sul) e à CAPES/PROCAD (Coordenação de Aperfeiçoamento de Pessoal de Nível Superior/Programa Nacional de Cooperação Acadêmica), 
Aceitação sensorial de bebidas lácteas potencialmente simbióticas

BURKERT, J. F. M. et al.

pelo apoio financeiro e pela concessão de Bolsa de Iniciação Científica.

\section{Referências}

ARYANA, K. J.; McGREW, P. Quality attributes of yogurt with Lactobacillus casei and various prebiotics. LWT - Food Science and Technology, London, v. 40, n. 10, p. 1808-1814, 2007. http:// dx.doi.org/10.1016/j.Iwt.2007.01.008

ASSOCIAÇÃO BRASILEIRA DE NORMAS TÉCNICAS - ABNT. NBR13088: Teste de Comparação Pareada em Análise Sensorial de Alimentos e Bebidas. São Paulo: ABNT, 1994a. 7 p.

ASSOCIAÇÃO BRASILEIRA DE NORMAS TÉCNICAS - ABNT. NBR13170: Teste de Ordenação em Análise Sensorial de Alimentos E Bebidas. São Paulo: ABNT, 1994b. 7 p.

ASSOCIAÇÃO BRASILEIRA DE NORMAS TÉCNICAS - ABNT. NBR13526: Teste De Comparação Múltipla em Análise Sensorial de Alimentos e Bebidas. São Paulo: ABNT, 1995. 9 p.

BORGES, K. C.; MEDEIROS, A. C. L.; CORREIA, R. T. P. logurte de leite de búfala sabor cajá (Spondias lutea I.): caracterização físico-química e aceitação sensorial entre indivíduos de 11 a 16 anos. Alimentos e Nutrição, Araraquara, v. 20, n. 2, p. 295-300, abr./jun, 2009.

BRASIL. Agência Nacional de Vigilância Sanitária - ANVISA. RDC $n^{\circ} 278$, de 22 de setembro de 2005. Aprova as categorias de Alimentos e Embalagens Dispensados e com Obrigatoriedade de Registro. Diário Oficial da República Federativa do Brasil, Brasília, DF, 23 set. 2005. Disponível em: <http://www.anvisa. gov.br/e-legis/>. Acesso em: ago. 2010.

BRASIL. Agência Nacional de Vigilância Sanitária (ANVISA). Resolução n. 2, de 7 de janeiro de 2002. Regulamento técnico de substâncias bioativas e probióticos isolados com alegação de propriedade funcional ou de saúde. Diário Oficial da República Federativa do Brasil, Brasília, DF, 09 jan. 2002. p. 192-193.

CASTRO, F. P.; CUNHA, T. M.; BARRETO, P. L. M.; AMBONI, R. D M. C.; PRUDÊNCIO, E. S. Effect of oligofructose incorporation on the properties of fermented probiotic lactic beverages. International Journal of Dairy Technology, Malden, v. 62 , n. 1, p. 68-74, 2008. http://dx.doi.org/10.1111/j.14710307.2008.00447.x

CASTRO, F. P.; CUNHA, T. M.; OGLIARI, P. J.; TEÓFILO, R. F.; FERREIRA, M. M. C.; PRUDÊNCIO, E. S. Influence of different content of cheese whey and oligofructose on the properties of fermented lactic beverages: Study using response surface methodology. LWT - Food Science and Technology, London, v. 42, n. 5, p. 993-997, 2009. http://dx.doi.org/10.1016/j. Iwt.2008.12.010

CHOCKCHAISAWASDEE, S.; ATHANASOPOULOS, V. I.; NIRANJAN, K.; RASTALL, R. A. Synthesis of galactooligosaccharide from lactose using $\beta$-galactosidase from Kluyveromyces lactis: studies on membrane-fitted bioreactors.
Biotechnology and Bioengineering, Hoboken, v. 89, n. 4, p. 434-443, 2005. http://dx.doi.org/10.1002/bit.20357

CRITTENDEN, R. G.; PLAYNE, M. J. Production, properties and applications of food-grade oligosaccharides. Trends in Food Science \& Technology, Amsterdan, v. 7, n. 11, p. 353-361, 1996. http://dx.doi.org/10.1016/S0924-2244(96)10038-8

CUNHA, T. M.; ILHA. E. C.; AMBONI, R. D. M. C.; BARRETO, P. L. M.; CASTRO, F. P.; PRUDÊNCIO, E. S. A influência do uso de soro de queijo e bactérias probióticas nas propriedades de bebidas lácteas fermentadas. Brazilian Journal of Food Technology, Campinas, v. 12, n. 1, p. 23-33, 2009. http://dx.doi. org/10.4260/BJFT2009200800004

GOMES, F. P. Curso de Estatística Experimental. 14. ed. Piracicaba: ESALQ, 2000. 477 p.

HASLER, C. M.; BLOCH, A. S.; THOMSON C. A.; ENRIONE, E.; MANNING, C. Position of the American Dietetic Association: Functional Foods. Journal of American Dietetic Association, Bethesda, v. 104, n. 5, p. 814-826, 2004. http://dx.doi. org/10.1016/j.jada.2004.03.015

INSTITUTO ADOLFO LUTZ - IAL. Métodos Químicos e Físicos para Análise de Alimentos. 3. ed. São Paulo: IAL, 1985. v. 1, 553 p. (Normas analíticas do Instituto Adolfo Lutz).

MARTINS, A. R.; BURKERT, C. A. V. Revisão: Galactooligossacarídeos (GOS) e seus efeitos prebióticos e bifidogênicos. Brazilian Journal of Food Technology, Campinas, v. 12, n. 3, p. 230-240, 2009. http://dx.doi.org/10.4260/BJFT2009800900017 MARX, S.; WINKLER, S.; HARTMEIER, W. Metabolization of $\beta$-(26)-linked fructose-oligosaccharides by different bifidobacteria. FEMS Microbiology Letters, Amsterdam, v. 182, n. 1, p. 163-169, 2000. http://dx.doi.org/10.1111/j.1574-6968.2000. tb08891.x

MAZOCHI, V.; MATOS JÚNIOR, F. E.; VAL, C. H.; DINIZ, D. N.; RESENDE, A. F.; NICOLI, J. R.; SILVA, A. M. Iogurte probiótico produzido com leite de cabra suplementado com Bifidobacterium spp. Arquivo Brasileiro de Medicina Veterinária e Zootecnia, Belo Horizonte, v. 62, n. 6, p.1484-1490, 2010.

MEILGAARD, M.; CIVILLE, G. V.; CARR, B. T. Sensory Evaluation Techniques. 3. ed. Boca Raton: CRC Press, 1999. 387 p.

MORAIS, A. S.; FINZER, J. R. D.; LIMAVERDE, J. R. Cristalização de ácido cítrico: otimização operacional. Brazilian Journal of Food Technology, Campinas, v. 11, n. 4, p. 313-321, 2008

OLIVEIRA, L. B.; JURKIEWICZ, C. H. Influência de inulina e goma acácia na viabilidade de bactérias probióticas em leite fermentado simbiótico. Brazilian Journal of Food Technology, Campinas, v. 12, n. 2, p. 138-144, 2009. http:// dx.doi.org/10.4260/BJFT20095808

OLSON, D. W.; ARYANA K. J. An excessively high Lactobacillus acidophilus inoculation level in yogurt lowers product quality during storage. LWT - Food Science and Technology, London, 
Aceitação sensorial de bebidas lácteas potencialmente simbióticas

BURKERT, J. F. M. et al.

v. 41, n. 5, p. 911-918, 2008. http://dx.doi.org/10.1016/j. Iwt.2007.05.017

PIANA, M. L.; PERSANO, L. O.; BENTABOL, A.; BRUNEAU, E.; BOGDANOV, S.; GUYOT, C. D. Sensory analysis applied to honey: state of the art. Apidologie, Avignon, v. 35, n. 1, p. S26-S37, 2004. http://dx.doi.org/10.1051/apido:2004048

QUEIROZ, M. B.; RAFFAINI, A. C. G. G.; FADINI, A. L. Efeito da adição do açúcar invertido e do tempo de batimento nas características físicas de marshmallow. Brazilian Journal of Food Technology, Campinas, v. 6, n. 2, p. 359-364, 2003.

QUEIROZ, M. I.; TREPTOW, R. O. Análise sensorial para avaliação da qualidade dos alimentos. Rio Grande: Editora da FURG, 2006. 266 p.

RODRIGUES, M. I.; IEMMA, A. F. Planejamento de Experimentos e Otimização de Processos. 3. ed. Campinas: Casa do espírito amigo fraternidade fé e amor, 2009. 358 p.

STONE, H.; SIDEL, J. L. Sensory Evaluation Practices. Orlando: Academic Press, 1985. 311 p.

TEIXEIRA, E. Análise Sensorial de Alimentos. Santa Catarina: UFSC, 1987. $119 \mathrm{p}$.
THAMER, K. G.; PENNA, A. L. B. Caracterização de bebidas lácteas funcionais fermentadas por probióticos e acrescidas de prebiótico. Ciência e Tecnologia de Alimentos, Campinas, v. 26, n. 3, p.589-595, 2006. http://dx.doi.org/10.1590/S010120612006000300017

VALIM, M. F.; ROSSI, E. A.; SILVA, R. S. F.; BORSATO, D. Sensory acceptance of a functional beverage based on orange juice and soymilk. Brazilian Journal of Food Technology, Campinas, v. 6, n. 2, p. 153-156, 2003.

VILELA, D. A importância econômica, social e nutricional do leite. Revista Batavo, Carambeí, n. 111, p. 03, 2002.

VILLEGAS, B.; TÁRREGA, A.; CARBONELL, I.; COSTELL, E. Optimising acceptability of new prebiotic low-fat milk beverages. Food Quality and Preference, Essex, v. 21, n. 2, p. 234-242, 2009. http://dx.doi.org/10.1016/j.foodqual.2009.03.001

VINDEROLA, C. G.; REINHEIMER, J. A. Culture media for the enumeration of Bifidobacterium bifidum and Lactobacillus acidophilus in the presence of yoghurt bacteria. International Dairy Journal, Edmonton, v. 9, n. 8, p. 497-505, 1999. http:// dx.doi.org/10.1016/S0958-6946(99)00120-X 